\title{
Assessment of Intermingled Phlegm and Blood Stasis Syndrome in Coronary Heart Disease: Development of a Diagnostic Scale
}

\author{
Xuan Zhou (D), Xian-tao Li ${ }^{D}$, Xiao-qi Liu, Bing Wang, and Ge Fang \\ School of Basic Medical Science, Guangzhou University of Chinese Medicine, Guangzhou 510006, China \\ Correspondence should be addressed to Xian-tao Li; 1xt150@126.com
}

Received 28 June 2018; Revised 26 September 2018; Accepted 9 October 2018; Published 24 October 2018

Academic Editor: Shan-Yu Su

Copyright (C) 2018 Xuan Zhou et al. This is an open access article distributed under the Creative Commons Attribution License, which permits unrestricted use, distribution, and reproduction in any medium, provided the original work is properly cited.

\begin{abstract}
Background. Intermingled Phlegm and Blood Stasis Syndrome (IPBSS) is a common feature in patients with coronary heart disease (CHD). In clinical practice, the diagnostic agreement of clinical doctor of Chinese Medicine (CM) is poor. We previously developed a IPBSS diagnostic scale for use by general practitioner. Objectives. To assess a IPBSS diagnostic scale that we previously developed for use by non-experts. Methods. This is a multicenter, prospective study involving eight study sites across China. Eligible patients were adults ( $\geq 18$ years) with $\mathrm{CHD}$ as demonstrated by a history of myocardial infarction, stenosis, or past coronary revascularization. IPBSS was assessed using a scale that consisted of 14 items in two domains (e.g., phlegm and blood stasis). The score range for each item was 0 to 3 points. Maximum total score was 72 points. Diagnostic accuracy was verified using consensus opinion by two independent experts as reference. Results. A total of 1,142 CHD patients were included. IPBSS was established in 729 subjects using the IPBSS diagnostic scale. In ROC curve analyses, at the optimal cut-off of 25.5 , the sensitivity and specificity of the IPBSS scale were $67.6 \%$ and $72.4 \%$, respectively. The area under the ROC curve was 0.741 (95\%CI: 0.711-0.772). Conclusions. The newly developed IPBSS scoring system showed moderate performance in diagnosing IPBSS in CHD patients. Data from further large-scale diagnostic test accuracy studies are warranted. This trial is registered with ChiCTR-OOC-15006599.
\end{abstract}

\section{Introduction}

Coronary heart disease (CHD) is one of the major causes of mortality and morbidity in developed countries [1]. Although CHD-related mortality has gradually declined over the last decades, it remains a leading cause of death in Europe [2]. In China, the number of people diagnosed with CHD has reached a total of 11 million in 2017 [3]. Many CHD patients have been successfully managed by CM, which emphasizes pattern identification [4]. Such an approach helps to identify the underlying pathological bases of the disease and is critical in selection of optimal treatment [5]. Effective integration of pattern identification from $\mathrm{CM}$ with differential diagnosis from Western medicine provides a solid basis to advancing the development of a model for individualized medicine [6].

The core value of pattern identification is CM syndrome, which is identified by experts through comprehensive analysis using four diagnostic methods that are unique to the practice of CM [7]. CM syndrome may evolve during disease progression. As a result, lack of simple and standardized assessment protocol represent a major obstacle in using pattern identification in daily medical practice. Efforts have been devoted to resolve this issue, including the use of hyperspectral medical tongue images for tongue diagnosis [8], tactile sensor system to detect pulse signals for pulse diagnosis [9], and scales and questionnaires developed to standardize disease diagnosis [10]. Scales and questionnaires could be divided into four types: "different disease with same syndrome" diagnostic scale of CM syndrome [11], "disease combined syndrome (multiple)" diagnostic scale of CM syndrome [12], Syndrome Factors Diagnostic scale [13], and "disease combined syndrome (single)" diagnostic scale of CM syndrome [14]. However, these diagnostic scales are typically developed without rigorous scientific grounds in item selection and often lack validation [15]. We have previously developed a diagnostic scale to assess IPBSS in CHD [16]. In the current study, we examined the performance of this scale in a group of $\mathrm{CHD}$ patients. 


\section{Methods}

2.1. Study Design. This is a multicenter, prospective diagnostic study involving eight study sites across China: Guangdong Provincial Hospital of Traditional Chinese Medicine, Hunan University of Traditional Chinese Medicine, Hubei Provincial Hospital of Traditional Chinese Medicine, Affiliated Hospital of Shandong University of Traditional Chinese Medicine, Guizhou Provincial Hospital of Traditional Chinese Medicine, Second Affiliated Hospital of Wenzhou Medical University, Changzhou City Hospital of Traditional Chinese Medicine, Tianjin University of Traditional Chinese Medicine. Enrolment commenced in October 2016 and ended in March 2018. Reporting of the results conforms to the Standards for Reporting of Diagnostic Accuracy (STARD) statement [17]. All study subjects provided written informed consent

\subsection{Participants}

2.2.1. Eligibility Criteria. Adult subjects ( $\geq 18$ years) suspected of having CHD based on the presence of at least one of the followings conditions were eligible for inclusion:

(1) history of myocardial infarction (ST-elevation myocardial infarction (STEMI) or non-ST-elevation myocardial infarction (NSTEMI));

(2) $>50 \%$ stenosis in at least one main branch of coronary arteries, as established by coronary angiography or computed tomography angiography;

(3) history of percutaneous coronary intervention (PCI) or coronary artery bypass grafting (CABG) $[18,19]$.

Subjects with unstable angina were excluded.

2.3. IPBSS Scale. The diagnostic scale for IPBSS was developed based on systematic literature review [20] of best available evidence via an expert consensus-based Delphi [21] and analytic hierarchy process (AHP) [22] approach. The scoring system included a total of 14 items [16] that could be classified domains into two domains (e.g., phlegm and blood stasis). Possible range of score was 0 to 3 points for each item and 0 to 72 points for the overall assessment.

After assessment using the IPBSS diagnostic scale, symptom differentiation was conducted independently by two CM experts with the following qualifications:

(i) specialization in cardiovascular diseases;

(ii) specialization in traditional Chinese medicine or integrative Chinese and Western medicine;

(iii) having a senior professional title;

(iv) engagement in daily clinical practice for at least 20 years.

Diagnosis of IPBSS was confirmed only on the basis where both experts reached agreement on a particular diagnostic case. If the two experts disagreed on patient status, IPBSS was considered not to be present. The performance of the IPBSS scoring system was investigated based on the consensus of opinion from the two experts. The two experts were not involved in assessing IPBSS using the scale.

2.4. Statistical Analysis. Statistical analysis was performed using the SPSS statistical software (IBM Corp. Released 2015. IBM SPSS Statistics for Windows, Version 23.0. Armonk, NY: IBM Corp.). Using the experts' diagnoses as the standard, we determined the receiver operating characteristic (ROC) curves for the IPBSS scores, the maximum Youden Index value, and the corresponding cut-off points [23]. In addition, the sensitivity, specificity, positive, and negative predictive values (PPV and NPV, respectively) were calculated using the following formulas:

(1) Sensitivity (true positive rate, TPR) $=$ [Patients diagnosed with IPBSS by the diagnostic scale / (Patients diagnosed with IPBSS + Patients with IPBSS but not diagnosed with IPBSS)] $\times 100 \%$.

(2) Specificity (true negative rate, TNR) $=$ [Patients diagnosed with IPBSS by the diagnostic scale / (Patients with IPBSS but not diagnosed with IPBSS + Patients without IPBSS)] $\times 100 \%$.

(3) $\mathrm{PPV}=[$ Patients diagnosed with IPBSS / Patients who were tested for IPBSS] $\times 100 \%$.

(4) NPV $=$ [Patients determined not to have IPBSS / Patients who were tested for IPBSS] $\times 100 \%$.

Sample size was calculated as described previously [16].

\section{Results}

3.1. Patient Baseline Characteristics. Between 1 October 2016 and 30 March 2018, a total of 1,158 eligible CHD patients were invited to participate. A total of 16 participants did not complete the IPBSS scoring. The final analysis included 1,142 subjects, with a median age of 68 years (range: $32-94$ years), of whom over half were males (Figure 1)

Of the 1,142 subjects, $799(70.0 \%)$ had hypertension (I10, ICD-10), 346 (30.3\%) had type 2 diabetes mellitus (T2DM, E11)), 173 (15.1\%) had hyperlipidemia (E78), 132 (11.6\%) had cerebral infarction (I63), 106 (9.23\%) had gastritis (K29), 90 (7.9\%) had arrhythmias(I49), 72 (6.3\%) had lung infection (J18), 56 (4.9\%) had chronic obstructive pulmonary disease (COPD, J44), 47 (4.1\%) had hyperuricemia (E79), and 47 (4.1\%) had atherosclerosis (I70) (Table 1).

3.2. Performance of the IPBSS Scale. The total score of IPBSS scale did not conform with normal distribution (median score: 21, range 12-33). Using the ROC analysis, the optimal cut-off score of the IPBSS diagnostic scale was determined to be 25.5 (Table 2). At this cut-off, the sensitivity, specificity, PPV, and NPV of the diagnostic scale were $67.6 \%, 72.4 \%$, $81.2 \%$, and $55.9 \%$, respectively. The area under the curve (AUC) was 0.741 (95\% confidence interval (CI), 0.711-0.772) (Figure 2). 
TABLE 1: Demographics of the study participants.

\begin{tabular}{|c|c|c|c|}
\hline & $\begin{array}{c}\text { IPBSS } \\
(\mathrm{n}=729)\end{array}$ & $\begin{array}{c}\text { Non-IPBSS } \\
(\mathrm{n}=413)\end{array}$ & $\begin{array}{l}\text { Overall population } \\
(\mathrm{n}=1142)\end{array}$ \\
\hline \multicolumn{4}{|l|}{ Gender } \\
\hline Male & $460(63.1)$ & $240(58.1)$ & $700(61.3)$ \\
\hline \multicolumn{4}{|l|}{ Age } \\
\hline$\leq 40$ & $8(1.1)$ & $2(0.5)$ & $10(0.9)$ \\
\hline$\leq 60$ & $176(24.1)$ & $85(20.6)$ & $261(22.9)$ \\
\hline$\leq 80$ & $472(64.8)$ & $263(63.6)$ & $735(64.4)$ \\
\hline$>80$ & $73(10)$ & $63(15.3)$ & $136(11.9)$ \\
\hline \multicolumn{4}{|l|}{ Co-morbidities } \\
\hline Hypertension & $518(71.1)$ & $281(68.03)$ & $799(70.0)$ \\
\hline $\mathrm{T} 2 \mathrm{DM}$ & $207(28.4)$ & $139(33.7)$ & $346(30.3)$ \\
\hline Hyperlipidemia & $113(15.5)$ & $60(14.5)$ & $173(15.1)$ \\
\hline Cerebral infarction & $70(9.6)$ & $62(15.0)$ & $132(11.6)$ \\
\hline Gastritis & $60(8.2)$ & $46(11.1)$ & $106(9.23)$ \\
\hline Arrhythmias & $48(6.6)$ & $42(10.2)$ & $90(7.9)$ \\
\hline Lung infection & $39(5.3)$ & $33(8.00)$ & $72(6.3)$ \\
\hline COPD & $35(4.8)$ & $21(5.08)$ & $56(4.9)$ \\
\hline Hyperuricemia & $29(4.0)$ & $18(4.36)$ & $47(4.1)$ \\
\hline Atherosclerosis & $26(3.6)$ & $21(5.08)$ & $47(4.1)$ \\
\hline
\end{tabular}

T2DM, type 2 diabetes mellitus; COPD, chronic obstructive pulmonary disease; IPBSS, Intermingled Phlegm and Blood Stasis Syndrome. All comorbidities were diagnosed according to the International Statistical Classification of Diseases and Related Health Problems 10th Revision (ICD-10).

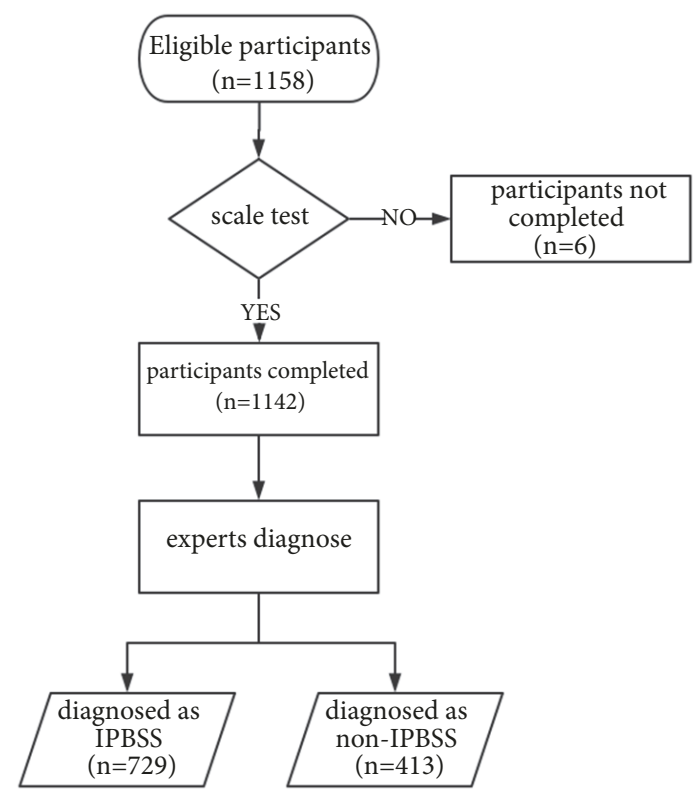

Figure 1: A flow diagram of the participants.

\section{Discussion}

The present study showed that, using a cut-off score of 25.5, our novel IPBSS scoring system produced reasonable sensitivity and specificity in differentiating patients with IPBSS from those without, using expert diagnoses as the reference.

Currently, only a few CM diagnostic scales are available [24], and some of these scales do not provide clear

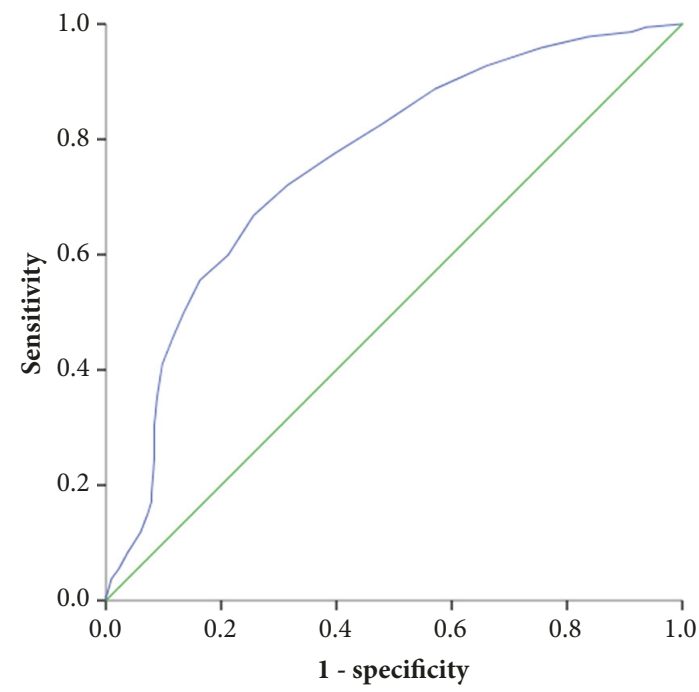

FIGURE 2: ROC analysis of the IPBSS diagnostic score (cut-off: 25.5) in assessing the presence versus absence of IPBSS. The area under the curve (AUC) was 0.741 .

explanation or description of the methodology of their development processes [12], and some without adequate quality control in scale design procedure, data entry [25]. Therefore, utilization of these diagnostic scales poses substantial issues in terms of consistency and accuracy.

Consequently, we conducted the current study to illustrate an effective validation method of a diagnostic scale by systematically reviewing the current literature together with a AHP-Delphi consensus method to establish the list 
TABLE 2: Performance of the IPBSS scoring system at various cut-off values.

\begin{tabular}{lccc}
\hline IPBSS score & Sensitivity & Specificity & Youden index \\
\hline 16.5 & 0.872 & 0.443 & 0.315 \\
17.5 & 0.844 & 0.479 & 0.323 \\
18.5 & 0.826 & 0.508 & 0.334 \\
19.5 & 0.802 & 0.554 & 0.356 \\
20.5 & 0.774 & 0.581 & 0.355 \\
21.5 & 0.745 & 0.608 & 0.353 \\
22.5 & 0.728 & 0.644 & 0.372 \\
23.5 & 0.713 & 0.678 & 0.391 \\
24.5 & 0.69 & 0.702 & 0.392 \\
$\mathbf{2 5 . 5}$ & $\underline{\mathbf{0 . 6 7 6}}$ & $\underline{\mathbf{0 . 7 2 4}}$ & $\mathbf{0 . 4 0 0}$ \\
26.5 & 0.646 & 0.741 & 0.387 \\
27.5 & 0.62 & 0.763 & 0.383 \\
28.5 & 0.583 & 0.794 & 0.377 \\
29.5 & 0.561 & 0.801 & 0.362 \\
30.5 & 0.523 & 0.809 & 0.332 \\
31.5 & 0.49 & 0.826 & 0.316 \\
\hline
\end{tabular}

of items to be included in the IPBSS diagnostic tool, eventually reaching a total of 14 items. The items we examined were extracted from modern standards and guidelines $[26,27]$, which were the result of wisdom by traditional Chinese medicine researchers. And the content validity of the scale depends on the quality of these standards and guidelines. Weighting of each item was achieved via the AHP. A four-tiered classification was designed to score each item ( 0 to 3 points), thereby providing a total score ranging from 0 to 72 points. We subsequently developed ROC curves and observed that a cut-off score of 25.5 was associated with optimal sensitivity, specificity, PPV, and NPV.

The current study has several limitations. First, we only assessed China-based participants and did not include nonCHD subjects [28]. Our study population is, therefore, not representative and whether our IPBSS scoring system could be used to assess subjects with other conditions of other ethnicities requires further investigation. Second, we prespecified opinion from two experts as the gold standard. A true "gold standard" is hard to attain in studies exploring diagnostic test accuracy of traditional Chinese medicine. Wang and Zhou proposed the use of random effects models to estimate the diagnostic performance without a gold standard by accounting for the correlation structure among different tests or practitioners [29]. However, the models proposed are in need of further investigation to fully validate their application. We opted to use the expert consensus method not only because this method is already widely used but also because it is highly unlikely for two experts to provide entirely different opinions in $\mathrm{CM}$ diagnoses, given that the experts we enrolled to participate were all strong candidates with solid theoretical knowledge and practical experience. Third, our diagnostic scale is yet to be verified using a large-scale, external study cohort. For future development, we anticipate to further modify the diagnostic scale with potentially less for ease of use.
In conclusion, our novel IPBSS scoring system produced moderate performance in identifying the presence of IPBSS in Chinese CHD patients. And we will improve the scoring system in future.

\section{Abbreviations}

IPBSS: Intermingled Phlegm and Blood Stasis Syndrome

CHD: Coronary heart disease

CM: $\quad$ Chinese medicine

AHP: Analytic hierarchy process

ACC: American College of Cardiology

AHA: American Heart Association

STEMI: ST-elevation myocardial infarction

NSTEMI: Non-ST-elevation myocardial infarction

PCI: Percutaneous coronary interventional

CABG: Coronary artery bypass grafting

CTA: Computed tomography angiography

ROC: Receiver operator characteristic

T2DM: Type 2 diabetes mellitus

COPD: Chronic obstructive pulmonary disease

AUC: Area under the curve.

\section{Data Availability}

The data used to support the findings of this study are available from the corresponding author upon request.

\section{Ethical Approval}

The current study was approved by the Medical Ethics Committee of Tianjin University of Traditional Chinese Medicine (no. TJUTCM-EC2015000).

\section{Conflicts of Interest}

The authors declare that they have no conflicts of interest. 


\section{Authors' Contributions}

Xian-tao Li conceptualized the study and contributed to manuscript drafting. Xian-tao Li and Xuan Zhou participated in study design and contributed to manuscript writing. Xiaoqi Liu, Bing Wang, and Ge Fang participated in the overall clinical research conduct and data analysis. All authors read and approved the final manuscript.

\section{Acknowledgments}

The authors thank the following teams for their valuable contributions: Yang Xiaobo Research Team of Guangdong Provincial Hospital of Traditional Chinese Medicine; Hu Zhixi Research Team of Hunan University of Traditional Chinese Medicine; Wu Bin Research Team of Hubei Provincial Hospital of Traditional Chinese Medicine; Dai Guohua Research Team of Affiliated Hospital of Shandong University of Chinese Medicine; Sun Gang Research Team of Guizhou Provincial Hospital of Traditional Chinese Medicine; Zheng Guoqing Research team of Second Affiliated Hospital of Wenzhou Medical University; Qian Weidong Research Team of Changzhou City Hospital of Traditional Chinese Medicine; Yu Chunquan Research Team of Tianjin University of Traditional Chinese Medicine. This study was supported by the National Basic Research Program of China (973 Program) (2014CB542901).

\section{References}

[1] M. Veerasamy, R. Edwards, G. Ford et al., "Acute coronary syndrome among older patients: A review," Cardiology in Review, vol. 23, no. 1, pp. 26-32, 2015.

[2] M. Nichols, N. Townsend, P. Scarborough, and M. Rayner, "Cardiovascular disease in Europe 2014: epidemiological update," European Heart Journal, vol. 35, no. 42, pp. 2929-2933, 2014.

[3] W. W. Chen, R. L. Gao, L. S. Liu et al., "Outline of report on cardiovascular diseases in China," Chinese Circulation Journal (Chinese), vol. 33, no. 1, pp. 1-8, 2017.

[4] S.-M. Li, H. Xu, and K.-J. Chen, "The diagnostic criteria of blood-stasis syndrome: considerations for standardization of pattern identification," Chinese Journal of Integrative Medicine, vol. 20, no. 7, pp. 483-489, 2014.

[5] A.-P. Lu and K.-J. Chen, "Integrative medicine in clinical practice: From pattern differentiation in traditional Chinese medicine to disease treatment," Chinese Journal of Integrative Medicine, vol. 15, no. 2, p. 152, 2009.

[6] J.-G. Li and H. Xu, "Incorporating pattern identification of chinese medicine into precision medicine: An integrative model for individualized medicine," Chinese Journal of Integrative Medicine, vol. 21, no. 11, pp. 807-810, 2015.

[7] F. W. Zhu and K. Z. Yuan, Diagnostics of Traditional Chinese Medicine, People's Medical Publishing House, Beijing, China, 2nd edition, 2011, edition.

[8] L. Zhi, D. Zhang, J.-Q. Yan, Q.-L. Li, and Q.-L. Tang, "Classification of hyperspectral medical tongue images for tongue diagnosis," Computerized Medical Imaging and Graphics, vol. 31, no. 8, pp. 672-678, 2007.

[9] Y.-F. Chung, C.-S. Hu, C.-C. Yeh, and C.-H. Luo, "How to standardize the pulse-taking method of traditional Chinese medicine pulse diagnosis," Computers in Biology and Medicine, vol. 43, no. 4, pp. 342-349, 2013.

[10] R. Okitsu, K. Iwasaki, Y. Monma et al., "Development of a questionnaire for the diagnosis of Qi stagnation," Complementary Therapies in Medicine, vol. 20, no. 4, pp. 207-217, 2012.

[11] S. Q. Wang, Q. Liu, S. N. Guo, L. Zhou, and Y. Gao, "Item selection analysis of qi deficiency and blood stasis evaluation questionnaire," Jilin Journal of Traditional Chinese Medicine (Chinese), vol. 35, no. 7, pp. 656-610, 2015.

[12] L. Wan, X. K. Huang, L. Wang, and Y. Yang, "The effects of fushengong formula on renal function and shh signal pathway of rats with chronic renal failure," Journal of Traditional Chinese Medicine (Chinese), vol. 56, pp. 1771-1774, 2015.

[13] K. Wang, Y. L. Lu, S. X. Tang, H. J. Li, Y. Z. Chen, and Y. Q. Ding, "Preliminary Research on Syndrome Factors Diagnostic Scale of Hypertensive Carotid Atherosclerosis," Chinese Archives of Traditional Chinese Medicine (Chinese), vol. 8, pp. 1863-1865, 2015.

[14] X. L. Zhang and Y. L. Li, “The development and test of diagnostic Scale item for Hypertension with kidney yang deficiency syndrome," Lishizhen Medicine \& Materia Medica Research (Chinese), vol. 26, no. 11, pp. 2797-2800, 2015.

[15] Q. Guo and Z. Chen, "Application of scale and questionaire in chinese syndrome research," Modern Journal of Integrated Traditional Chinese and Western Medicine (Chinese), vol. 21, no. 25, pp. 2847-2848, 2012.

[16] X. Liu, D. Peng, Y. Wang et al., "Diagnostic accuracy of chinese medicine diagnosis scale of phlegm and blood stasis syndrome in coronary heart disease: a study protocol," Chinese Journal of Integrative Medicine, pp. 1-6, 2018.

[17] J. F. Cohen, D. A. Korevaar, D. G. Altman et al., "STARD 2015 guidelines for reporting diagnostic accuracy studies: explanation and elaboration," BMJ Open, vol. 6, no. 11, p. e012799, 2016.

[18] G. N. Levine, E. R. Bates, J. A. Bittl et al., "ACC/AHA Guideline Focused Update on Duration of Dual Antiplatelet Therapy in Patients With Coronary Artery Disease: A Report of the American College of Cardiology /American Heart Association Task Force on Clinical Practice Guidelines," Journal of the American College of Cardiology, vol. 68, no. 10, pp. 243-1275, 2016.

[19] Chinese Society Of Cardiology., C. M. A., and C. J. O. C. Editorial Board, "Guideline for diagnosis and treatment of patients with chronic stable angina," Chinese Journal of Cardiology (Chinese), vol. 35, no. 3, pp. 195-206, 2007.

[20] R. Xie, D. Peng, Y. Wang et al., "Analysis of Research Documents on Syndrome Differentiation Standards," Journal of Guangzhou University of Traditional Chinese Medicine (Chinese), vol. 32, no. 02, pp. 356-360, 2015.

[21] R. Xie, D. Peng, Y. Wang et al., "Discussion on the basis of syndrome differentiation of TCM phlegm syndrome based on Delphi method," Liaoning Journal of Traditional Chinese Medicine (Chinese), vol. 44, no. 01, pp. 16-20, 2017.

[22] R. Xie, X. Lian, D. Peng et al., "Evaluation of the importance of syndrome differentiation basis of phlegm syndrome in coronary heart disease based on analytic hierarchy process," Lishizhen Medicine and Materia Medica Research (Chinese), vol. 28, no. 09, pp. 2301-2304, 2017.

[23] K. Hajian-Tilaki, "Receiver operating characteristic (ROC) curve analysis for medical diagnostic test evaluation," Caspian Journal of Internal Medicine, vol. 4, no. 2, pp. 627-635, 2013.

[24] Y. J. Sun, X. Chen, and Z. D. Yin, "A Literature Review on the Diagnosed Scale of Chinese Medicine," Guiding Journal of 
Traditional Chinese Medicine Pharmacy (Chinese), vol. 23, no. 16, pp. 111-114, 2017.

[25] W. L. Chen, J. Lu, W. Li, Z. J. Li, and Q. K. Wu, "Analysis of TCM Syndrome Scale of Chronic Hepatitis C," Journal of Shanxi College of Traditional Chinese Medicine (Chinese), vol. 11, no. 01, pp. 33-35, 2010.

[26] R. Xie, "Assessing for syndrome Differentiation Basis of Phlegm Syndrome Based on Delphi Method," Liaoning Journal of Traditional Chinese Medicine (Chinese), vol. 44, no. 1, pp. 16-20, 2017.

[27] F. Duan, Methodology and Macroscopic Diagnostic Research on Criteria of Coronary Heart Disease with Intermingled Phlegm and Blood Stasis Syndrome, Hubei University of Traditional Chinese Medicine, Wu Han, 2017.

[28] M. J. Lv, The Development and evaluation of the scale of stable angina (the syndrome of phlegm and blood stasis in TCM), Liaoning University of Traditional Chinese Medicine, Shen Yang, 2014.

[29] Z. Wang and X.-H. Zhou, "Random effects models for assessing diagnostic accuracy of traditional Chinese doctors in absence of a gold standard," Statistics in Medicine, vol. 31, no. 7, pp. 661-671, 2012. 


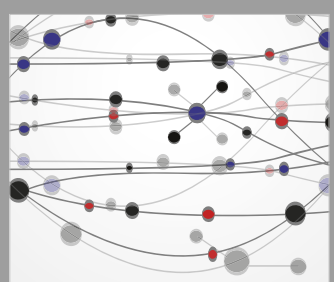

The Scientific World Journal
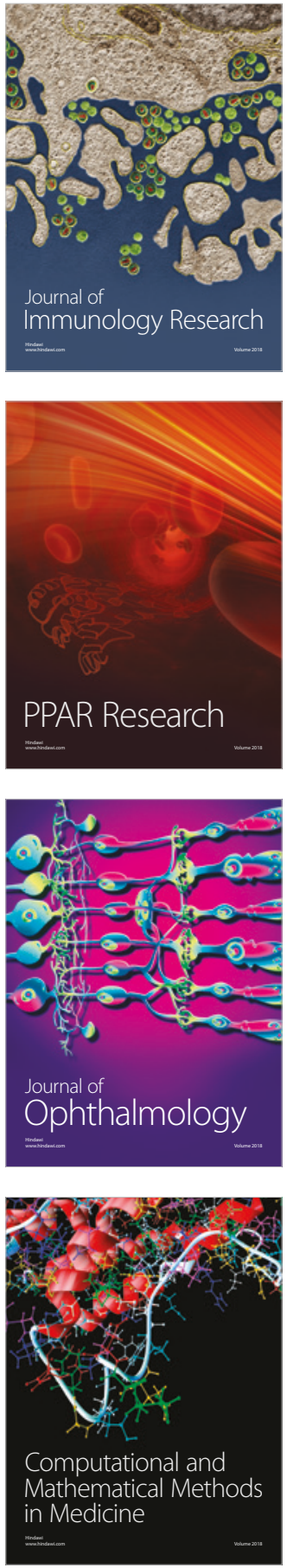

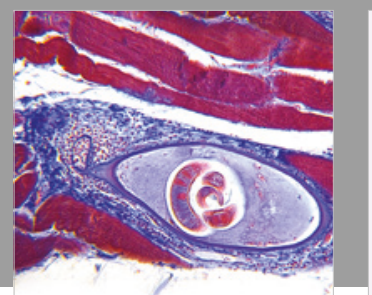

Gastroenterology Research and Practice

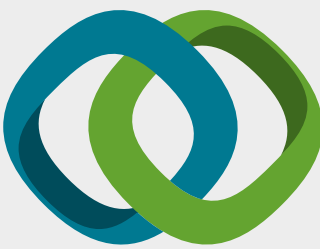

\section{Hindawi}

Submit your manuscripts at

www.hindawi.com
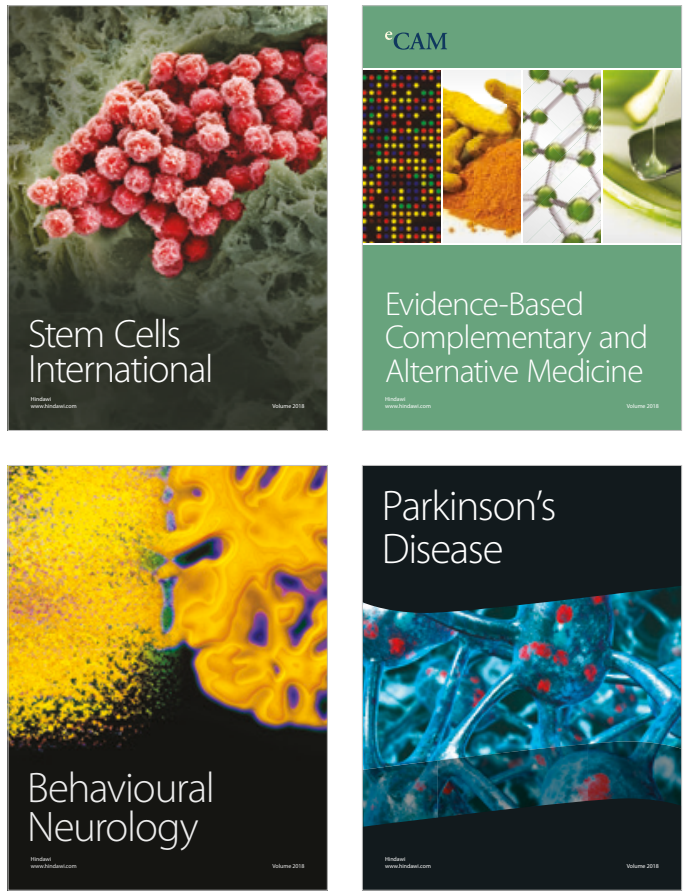

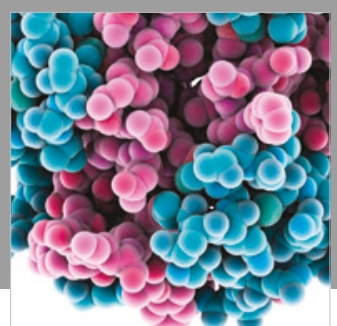

ournal of

Diabetes Research

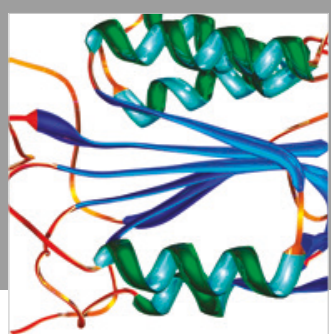

Disease Markers
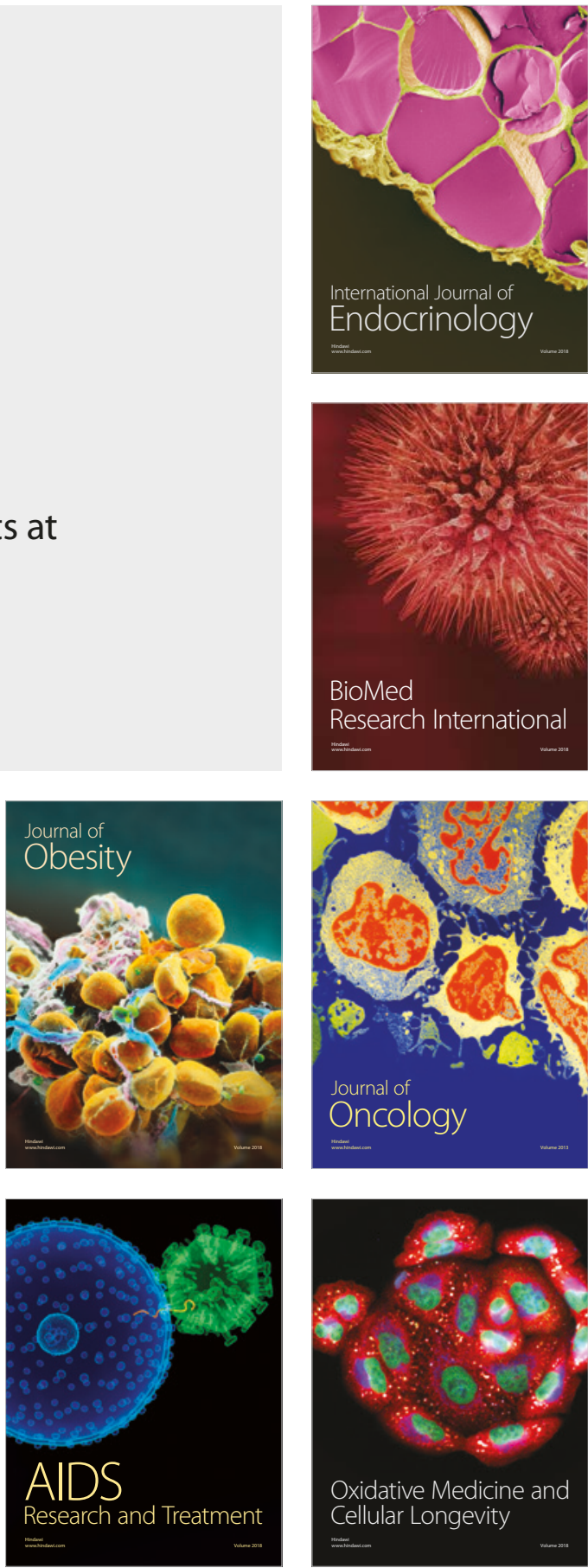